City University of New York (CUNY)

CUNY Academic Works

\title{
An Early Assessment of Medium Range Monsoon Precipitation Forecasts from the Latest High-Resolution NCEP-GFS (T1534) Model over South Asia
}

\author{
Satya Prakash \\ CUNY New York City College of Technology \\ Imaranali M. Momin \\ National Centre for Medium Range Weather Forecasting, ESSO/MoES \\ Ashis K. Mitra \\ National Centre for Medium Range Weather Forecasting, ESSO/MoES \\ Partha S. Bhattacharjee \\ NOAA/NWS National Centers for Environmental Prediction \\ Fanglin Yang \\ NOAA/NWS National Centers for Environmental Prediction
}

See next page for additional authors

\section{How does access to this work benefit you? Let us know!}

More information about this work at: https://academicworks.cuny.edu/ny_pubs/74

Discover additional works at: https://academicworks.cuny.edu

This work is made publicly available by the City University of New York (CUNY).

Contact: AcademicWorks@cuny.edu 


\section{Authors}

Satya Prakash, Imaranali M. Momin, Ashis K. Mitra, Partha S. Bhattacharjee, Fanglin Yang, and Vijay Tallapragada 


\title{
An Early Assessment of Medium Range Monsoon Precipitation Forecasts from the Latest High-Resolution NCEP-GFS (T1534) Model over South Asia
}

\author{
Satya Prakash ${ }^{1 *}$, Imranali M. Momin ${ }^{2}$, Ashis K. Mitra ${ }^{2}$, Partha S. BhattacharJeE ${ }^{3}$, \\ FANGLIN YANG ${ }^{3}$, and VIJAY TALLAPRAGADA ${ }^{4}$ \\ ${ }^{1}$ New York City College of Technology, \\ The City University of New York, Brooklyn, NY, USA \\ *E-mail: spsharma_01@yahoo.co.in; sprakash@ citytech.cuny.edu
${ }^{2}$ National Centre for Medium Range Weather Forecasting, ESSO/MoES, Noida, UP, India

${ }^{3}$ I. M. Systems Group, Inc., NOAA/NWS National Centers for Environmental Prediction, College Park, MD, USA

${ }^{4}$ NOAA/NWS National Centers for Environmental Prediction, College Park, MD, USA

Pure and Applied Geophysics (2016) Vol. 173, pp. 2215-2225, doi:10.1007/s00024-016-1248-5. 


\begin{abstract}
Reliable prediction of the South Asian monsoon rainfall and its variability is crucial for various hydrological applications and early warning systems. The National Centers for Environmental Prediction - Global Forecast System (NCEP-GFS) is one of the popular global deterministic numerical weather prediction models, which is recently upgraded from T574 to T1534. In this paper, medium range monsoon precipitation forecasts from both the T1534 and T574 models are critically evaluated over the South Asia for the peak monsoon months (July and August) of 2015. Although both the versions of GFS model show similar large-scale monsoon rainfall patterns, the dry bias over the northwest India and equatorial Indian Ocean is noticeably improved in day-1 through day-5 forecasts in the new high-resolution T1534 model. The error decomposition analysis shows similar error characteristics in the monsoon rainfall prediction from both the versions of GFS model, in general. However, forecast improvement factor shows 10-30\% improvement in precipitation forecast from the latest T1534 model over most parts of the South Asia. These preliminary analyses suggest that a suitable bias-correction to the GFS model precipitation forecasts will be useful for any specific application.
\end{abstract}

Key words: Numerical weather prediction, South Asian monsoon rainfall, Medium range weather forecast, Error decomposition, Forecast improvement factor. 


\section{Introduction}

The southwest monsoon rainfall spanning from June to September provides about threefourth of the annual rainfall over the South Asia. Variations in the monsoon rainfall at any spatiotemporal scale have paramount socio-economic impacts. Hence, reliable prediction of the South Asian monsoon rainfall and its variability is crucial (RAJEEVAN et al. 2010; TURNER and AnNamalai 2012; Collins et al. 2013; Webster 2013; PraKash et al. 2014). Floods and droughts associated with the monsoon rainfall cause major devastation in the South Asian region almost every year. Reliable prediction of the South Asian monsoon rainfall from the numerical weather prediction (NWP) models is vital for agriculture, water management, and early warning systems associated with extreme weather conditions. But, accurate prediction of the South Asian monsoon rainfall and its variability is still a formidable challenge due to its complex coupled atmosphere-ocean-land-ice interactive nature (MITRA et al. 2011, 2013; GADGIL and SRINIVASAN 2013). However, skill of recent NWP models is considerably improving in simulation and prediction of the South Asian monsoon rainfall due to the consistent improvement in models, observations and data assimilation techniques (HONG and DUDHIA 2012; MAGNUSSON and KALLEN 2013; RANADE et al. 2014; BAUER et al. 2015).

Among several state-of-the-art global deterministic NWP models operational at lead modeling centers worldwide, the National Centers for Environmental Prediction - Global Forecast System (NCEP-GFS; HAN and PAN 2011) is one of the widely used models for various applications. NCEP-GFS is a global spectral model in hybrid sigma-pressure vertical coordinates. In January 2015, the existing GFS T574 model was upgraded to T1534 at the NCEP. The latest T1534 version is supposed to be better than T574 version for precipitation forecasting in medium range due to major upgradations in physics, dynamics, resolution, and data 
assimilation technique. Recently, DORIAN and YANG (2015) evaluated precipitation forecasts from both the versions of GFS model over the contiguous United States using object-based diagnostic method and showed that the recent T1534 model had reduced bias than T574 model. Reliable high-resolution medium range precipitation forecasts would be very useful for hydrological applications and early warning systems. In this study, day-1 through day-5 precipitation forecasts from both the versions of GFS model (T1534 and T574) are comprehensively assessed over the South Asia for the peak southwest monsoon season (01 July 31 August) of 2015.

\section{Data and Methods}

\subsection{GSMaP Precipitation Data}

The Global Satellite Mapping of Precipitation (GSMaP) data sets are high-resolution multi-satellite precipitation estimates, which combine a variety of contemporary microwave imagers/sounders and infrared satellite observations (USHIO et al. 2009). GSMaP precipitation estimates are available in both near-real-time and delayed mode. Recently, a new algorithm for the Global Precipitation Measurement (GPM) mission has been implemented to retrieve precipitation and an advanced version of GSMaP precipitation (version 6) estimates is released in late 2014. The gauge-calibrated hourly GSMaP (GSMaP_Gauge) version 6 precipitation estimates available at $0.1^{\circ}$ latitude $\times 0.1^{\circ}$ longitude resolution from the Earth Observation Research Center, Japan Aerospace Exploration Agency (EORC/JAXA) are used in this study. The global daily gauge analyses based on the Climate Prediction Center (CPC) are used for biascorrection in the GSMaP estimates using the optimal theory (USHIO et al. 2013). This gauge- 
adjusted near-real-time precipitation product is reasonable over the Indian monsoon region (PRAKASH et al. 2016) and hence chosen as reference data set in this study.

\subsection{GFS Model Precipitation Forecast Data}

Precipitation forecasts from two distinct versions of the GFS model (T1534 and T574) are used in this study. NCEP-GFS model has 64 hybrid sigma-pressure levels and provides outputs in a Gaussian grid (HAN and PAN 2011). T574 $(\sim 27 \mathrm{~km})$ is also operational at the National Centre for Medium Range Weather Forecasting (NCMRWF), India since late 2010 (PRASAD et al. 2014). GFS model has split physics and dynamics, and uses leapfrog time integration scheme for non-linear advection terms and semi-implicit time integration scheme for gravity waves and zonal advection of moisture and vorticity. A detailed description of the model can be found at http://www.emc.ncep.noaa.gov/GFS/doc.php. In January 2015, the NCEP upgraded the GFS analysis and forecast system from T574 $(\sim 27 \mathrm{~km})$ to T1534 ( 13 km). There are several changes (e.g., initial conditions, physics, dynamics, data assimilation methods, etc.) made in the latest T1534 as compared to T574 despite of increased horizontal resolution alone. The Eulerian dynamics in T574 version is now replaced with the Semi-Lagrangian dynamics in T1534 model. Moreover, Gridpoint Statistical Interpolation (GSI) data assimilation method in T574 model is replaced with the Ensemble Kalman Filter (EnKF) data assimilation method in the recent T1534 model. More details about the changes can be found at http://www.nco.ncep.noaa.gov/pmb/changes/. Six-hourly precipitation forecasts at 0000 UTC initial condition from T1534 and T574 models for July and August 2015 are obtained from the NCEP and NCMRWF, respectively. It is also to be noted that there are some differences between NCEP and NCMRWF T574 models as discussed in detail by PRASAD et al. (2014). We believe 
that the T574 model run at the NCMRWF is dedicated to improve precipitation forecasts over the South Asian monsoon region. A similar version of the T574 model is also used in the India Meteorological Department; hence this study will indicate the benefits to Indian users from the updated version of the GFS model.

\subsection{Methodology}

As the observation and model precipitations are available at distinct horizontal resolutions, all the three precipitation data sets are re-projected at a same regular $0.25^{\circ}$ latitude/longitude grid using bilinear interpolation for comparison. Daily precipitation forecasts from both the versions of GFS model (T1534 and T574) are computed from six-hourly forecasts. To assess the capability of daily GFS precipitation forecasts, mean, bias, and mean-square error (MSE) are computed against observation for the study period. Moreover, the total MSE in GFS models are decomposed into two parts: (a) MSE due to mean difference and (b) MSE due to pattern variation. Both the components of the MSE depend on standard deviation and anomalous pattern correlation. These MSE components (YANG 2015) can be decomposed as:

$$
\begin{aligned}
& E^{2}=(\bar{F}-\bar{O})^{2}+\sigma_{F}^{2}+\sigma_{O}^{2}-2 \sigma_{F} \sigma_{O} R \\
& \text { with } R=\frac{1}{n} \sum_{\bar{i}=1}^{n}\left(F_{i}-\bar{F}\right)\left(O_{i}-\bar{O}\right) /\left(\sigma_{F} \sigma_{O}\right)
\end{aligned}
$$

where, $n$ is the number of matches, $\sigma$ is standard deviation, and the subscripts $F$ and $O$ stand for model and reference precipitation, respectively. First term in right hand side of equation (1) is the MSE by mean differences and the last three terms altogether be MSE by pattern variations. 
In order to assess the potential of latest T1534 precipitation forecasts with respect to T574, forecast improvement factor (FIF) is computed for each day forecast. FIF is computed in percentage following DAS et al. (2015):

$$
F I F=\left(\frac{\text { RMSE }_{T 574}-\text { RMSE }_{T 1584}}{\text { RMSE }_{T 574}}\right) \times 100 \%
$$

Where, $R M S E_{T 574}$ and $R M S E_{T 1534}$ are centered root-mean-square error (RMSE) of T574 and T1534 with respect to observation, respectively. A positive value of FIF indicates an improvement in T1534 version over T574, whereas a negative FIF indicates degradation of precipitation forecast in the recent high-resolution GFS model. Furthermore, an equitable skill score - Peirce Skill Score (PSS) and frequency bias index (FBI) based on a $2 \times 2$ contingency table (WILKS 2006; HoGAN et al. 2010) given by equations (4) and (5), respectively are also computed for different rainfall thresholds to assess the skill of the precipitation forecasts over the study region.

$$
\begin{gathered}
\text { PSS }=\frac{\text { hits }}{\text { (hits }+ \text { misses })}-\frac{\text { false alarms }}{(\text { false alarms }+ \text { correct rejects })} \\
F B I=\frac{(\text { hits }+ \text { false alarms })}{(\text { hits }+ \text { misses })}
\end{gathered}
$$

\section{Results and Discussions}

Fig. 1 shows the mean monsoon rainfall for the study period from observed as well as model data sets. Daily forecasts from both the versions of GFS model are computed from six- 
hourly forecasts and then averaged for July-August 2015 (i.e., 62 days) to compute mean rainfall. All the analyses in this paper are done for day- 1 through day- 5 forecasts, but the results of day-1, day-3 and day-5 forecasts are shown throughout for brevity. Additionally, the terms 'precipitation' and 'rainfall' are interchangeably used. The well-known large-scale monsoon rainfall patterns are similar in both the versions of GFS models. Lower rainfall belt separating high rainfall regimes of central India and the Himalayan foothills region is notably improved in T1534 than T574 forecasts. Lower rainfall over the northwest India also shows an improvement in the new version model as compared to the observation. To assess the quantitative difference from the observation, biases in both T1534 and T574 precipitation forecasts are computed for the study period, which are shown in Fig. 2. In general, GFS model exhibits wet bias over the eastern Arabian Sea (AS), southern Bay of Bengal (BOB) and Myanmar regions, whereas it shows dry bias over the northern BOB, equatorial India Ocean (EIO) and northwest India. The dry bias over the northwest India and EIO is notably improved in all the day forecasts from the latest GFS model. But, the regions having wet bias do not show any improvement in T1534 model.

The temporal correlation between model and observed precipitation for the study period is presented in Fig. 3. The latest high-resolution GFS model shows an improvement over T574 in northwest and central India across the length of forecasts. Fig. 4 shows the spatial distributions of MSE in both T1534 and T574 precipitation forecasts as compared to the observation. MSE shows similar characteristics over most parts of the study region in both the versions of GFS model. There is no any drastic improvement in MSE in the latest high-resolution GFS model. As expected, higher mean rainfall regions have larger MSE and lower mean rainfall regimes have smaller MSE. However, MSE over the west India around Gujarat is larger for T1534 compared 
to T574 for day-1 and day-3 forecasts, which might be due to increase in wet bias in T1534 model (Fig. 2). The components of MSE, presented in percentage, for both the versions of GFS models are illustrated in Figs. 5 and 6, respectively. Most parts of the study region have larger MSE due to pattern variations than MSE by mean differences in both T1534 and T574 models. The southern BOB and the AS have larger MSE by mean differences in both the versions of GFS model.

Fig. 7 presents FIF in the latest high-resolution GFS model. Interestingly, the latest highresolution GFS model shows 10-30\% improvement over T574 model in precipitation forecast over most parts of the South Asia. But, the forecast is degraded in day-5 over the western part of the study region in T1534. The bias structure looks like wet bias especially over western part of India is slightly increased in the T1534 (Fig. 2), which is in agreement with Fig. 7 that, FIF is mainly negative over these region. The improvement in precipitation forecast is larger at land regions than the ocean regions. The overall improvement in T1534 can be attributed to better physics, improved initialization and data assimilation rather than resolution alone. Recently, SAHAI et al. (2015) using a coupled model showed that increasing model resolution alone without attempting any modifications in physics would not improve the extended range prediction over the Indian monsoon region. The variations in the PSS and FBI in both the versions of GFS model for different daily precipitation thresholds over the study region are presented in Fig. 8. The new high-resolution model shows marginally better PSS than T574 in day-1 through day-5 forecasts. However, FBI is larger in T1534 than T574. In general, PSS decreases with the increase of precipitation threshold as well as with forecast length. Similar decrease in FBI is also observed for T1534 model, but not for T574 model. 
The monsoon rainfall over the core monsoon zone of India bounded by $18^{\circ} \mathrm{N}-28^{\circ} \mathrm{N}$ and $65^{\circ} \mathrm{E}-88^{\circ} \mathrm{E}$ determines active and break spells of monsoon across the country (RAJEEVAN et al. 2010), which is crucial for agricultural sectors. The time-series of domain-mean rainfall over the core monsoon zone from observation and model data sets for the study period are shown in Fig. 9(a). The latest GFS model is able to pick up the peaks of rainfall better than T574 model up to day-3 forecasts. A phase shift appears in the GFS model precipitation forecasts over this region as compared to the observation. Fig. 9(b) presents the probability distribution functions (PDF) of monsoon precipitation over the core monsoon zone from observation and model data sets for the study period. It can be noted that both the versions of GFS model largely overpredicts light rainfall of less than $2 \mathrm{~mm} \mathrm{day}^{-1}$ over the core monsoon zone. DORIAN and YANG (2015) also reported similar results over the contiguous United States using object-based evaluation method. The PDF of the model rainfall are in good agreement with observation for higher rainfall ranges, and T1534 shows a marginal improvement over T574 model.

\section{Conclusions}

The variations in the intensity, frequency and duration of the southwest monsoon rainfall over the South Asia have significant socio-economic impacts. Reliable simulation and prediction of the monsoon rainfall is very important for various sectors like hydrology, agriculture, and disaster preparedness. NCEP-GFS is one of the popular global deterministic NWP models, which is upgraded from T574 to T1534 in January 2015. In addition to improvement in horizontal resolution and initial conditions, several physical and dynamical changes were also incorporated in the new version. In this study, day-1 through day-5 South Asian monsoon precipitation forecasts from both the versions of GFS model (NCEP-T1534 and NCMRWF- 
T574) were assessed for the months of July and August 2015. Even though both the versions of GFS model showed similar large-scale monsoon rainfall patterns for the study period, the dry bias over the northwest India and EIO was considerably improved throughout the length of forecasts from day-1 through day-5 in the latest T1534 model. The MSE and error decomposition analysis showed overall similar error characteristics in the South Asian monsoon rainfall prediction from both the versions of GFS model. However, FIF showed 10-30\% improvement in precipitation forecast from T1534 model over most parts of the South Asia. Additionally, T1534 showed marginal improvement over T574 in predicting higher monsoon rainfall over the core monsoon zone. Overall this preliminary analysis using two months of data suggests that a suitable bias-correction is needed in the GFS model precipitation forecasts before its integration to any specific application. However, we plan to extend this study for a longer time period along with associated atmospheric variables to better understand the cause of precipitation difference in both the versions of GFS model. Nevertheless, there is a great need of further advancement in model physics, dynamics, parameterization schemes, etc. to predict the South Asian monsoon rainfall more reliably in medium range for various applications in nearreal-time.

Acknowledgements: The authors would like to thank the editor-in-chief and three anonymous reviewers for their constructive comments. The GSMaP precipitation data obtained from the EORC/JAXA, GFS T1534 and T574 precipitation forecast data obtained from NCEP/EMC Monsoon Desk and NCMRWF, respectively are thankfully acknowledged.

\section{REFERENCES}

BAuer, P., Thorpe, A., and Brunet, G., (2015), The quiet revolution of numerical weather prediction, Nature, 525, 47-55, doi:10.1038/nature14956.

Collins, M., Achutarao, K., Ashok, K., Bhandari, S., Mitra, A.K, Prakash, S., SRIVAStAVA, R., and TURNer, A., (2013), Observational challenges in evaluating climate models, Nat. Clim. Change, 3, 940-941, doi:10.1038/nclimate2012. 
DAs, S.K., DeB, S.K., KishTawal, C.M., and PAL, P.K., (2015), Validation of seasonal forecast of Indian summer monsoon rainfall, Pure Appl. Geophys., 172, 1699-1716, doi:10.1007/s00024014-1024-3.

DORIAN, T.A., and YANG, F., (2015), Application of MODE to GFS (T574) and GFS (T1534) precipitation verification, $27^{\text {th }}$ Conference on Weather Analysis and Forecasting/23 ${ }^{\text {rd }}$ Conference on Numerical Weather Prediction, Chicago, USA.

Gadgil, S., and SRINIVASAn, J., (2013), Monsoon prediction: Hits, misses and false alarms, Curr. Sci., 104(8), 1005.

HAN, J., and PAN, H.-L., (2011), Revision of convection and vertical diffusion schemes in the NCEP Global Forecast System, Wea. Forecasting, 26, 520-533.

Hogan, R.J., Ferro, C.A.T., JollifFe, I.T., and StePhenson, D.B., (2010), Equitability revisited: Why the "equitable threat score" is not equitable, Wea. Forecasting, 25, 710-726, doi:10.1175/2009WAF2222350.1.

Hong, S.Y., and DudHIA, J., (2012), Next-generation numerical weather prediction: Bridging parameterization, explicit clouds, and large eddies, Bull. Amer. Meteor. Soc., 93, ES6-ES9, doi:10.1175/2011BAMS3224.1.

MAGnusson, L., and KALLEN, E. (2013), Factors influencing skill improvements in the ECMWF forecasting system, Mon. Wea. Rev., 141, 3142-3153, doi:10.1175/MWR-D-12-00318.1.

Mitra, A.K., Rajagopal, E.N., Iyengar, G.R., Mahapatra, D.K., Momin, I.M., Gera, A., Sharma, K., George, J.P., Ashrit, R., Dasgupta, M., Mohandas, S., Prasad, V.S., Basu, S., Arribas, A., Milton, S.F., Martin, G.M., Barker, D., and Martin, M., (2013), Prediction of monsoon using a seamless coupled modelling system, Curr. Sci., 104, 1369-1379.

Mitra, A.K., Iyengar, G.R., Durai, V.R., SAnjay, J., Krishnamurti, T.N., Mishra, A., and SIKKA, D.R., (2011), Experimental real-time multi-model ensemble (MME) prediction of rainfall during monsoon 2008: Large-scale medium-range aspects, J. Earth Syst. Sci., 120, 27-52, doi:10.1007/s12040-011-0013-5.

Prakash, S., Mitra, A.K., Aghakouchak, A., Liu, Z., Norouzi, H., and Pai, D.S., (2016), A preliminary assessment of GPM-based multi-satellite precipitation estimates over a monsoon dominated region, J. Hydrol., doi:10.1016/j.jhydrol.2016.01.029.

Prakash, S., Mitra, A.K., Momin, I.M., Rajagopal, E.N., and Basu, S., (2014), Agreement between monthly land rainfall estimates from TRMM-PR and gauge-based observations over South Asia, Remote Sens. Lett., 5(6), 558-567, doi:10.1080/2150704X.2014.934401 
Prasad, V.S., Mohandas, S., Dutta, S.K., Dasgupta, M., IYengar, G.R., Rajagopal, E.N., and BASU, S., (2014), Improvements in medium range weather forecasting system of India, J. Earth Syst. Sci., 123, 247-258.

RAJEEVAn, M., GAdGil, S., and BHATE, J., (2010), Active and break spells of the Indian summer monsoon, J. Earth Syst. Sci., 119(3), 229-247.

Ranade, A., Mitra, A.K., Singh, N., and BAsu, S., (2014), A verification of spatio-temporal monsoon rainfall variability across Indian region using NWP model output, Meteorol. Atmos. Phys., 125, 43-61, doi:10.1007/s00703-014-0317-5.

Sahai, A.K., Abhilash, S., Chattopadhyay, R., Borah, N., Joseph, S., Sharmila, S., and RAJEEVAN, M., (2015), High-resolution operational monsoon forecasts: An objective assessment, Clim. Dyn., 44, 3129-3140, doi:10.1007/s00382-014-2210-9.

Turner, A., and Annamalai, H., (2012), Climate change and the South Asian monsoon, Nat. Clim. Change, 2, 587-595, doi:10.1038/nclimate1495.

Ushio, T., Kubota, T., Shige, S., Okamoto, K., Aonashi, K., Inoue, T., Takahashi, N., Iguchi, T., Kachi, M., OKI, R., Morimoto, T., and Kawasaki, Z., (2009), A Kalman filter approach to the Global Satellite Mapping of Precipitation (GSMaP) from combined passive microwave and infrared radiometric data, J. Meteor. Soc. Japan, 87A, 137-151.

Ushio, T., Tashima, T., Kubota, T., and Kachi, M., (2013), Gauge adjusted Global Satellite Mapping of Precipitation (GSMaP_Gauge), Proc. 29th ISTS, 2013-n-48.

WeBsteR, P.J., (2013), Meteorology: Improve weather forecasts for the developing world, Nature, 493, 17-19, doi:10.1038/493017a.

WILKS, D.S., (2006), Statistical methods in the Atmospheric Sciences, $2^{\text {nd }}$ edition, Academic Press, Oxford, UK, 648 pp.

YANG, F., (2015), Decomposition of model forecast errors: Methodology and application, AGU Fall Meeting, 14-18 December 2015, San Francisco, USA. 


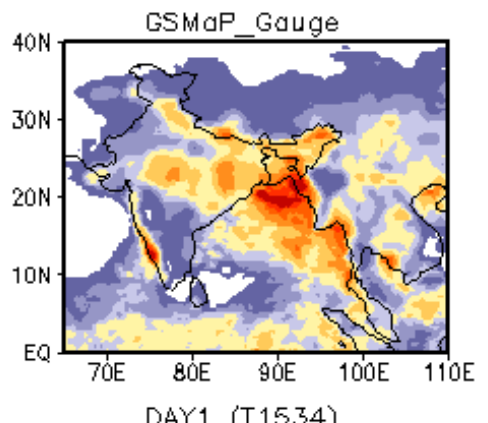

$$
\text { Mean Jul-Aug } 2015 \text { rain [mm day-1 }
$$
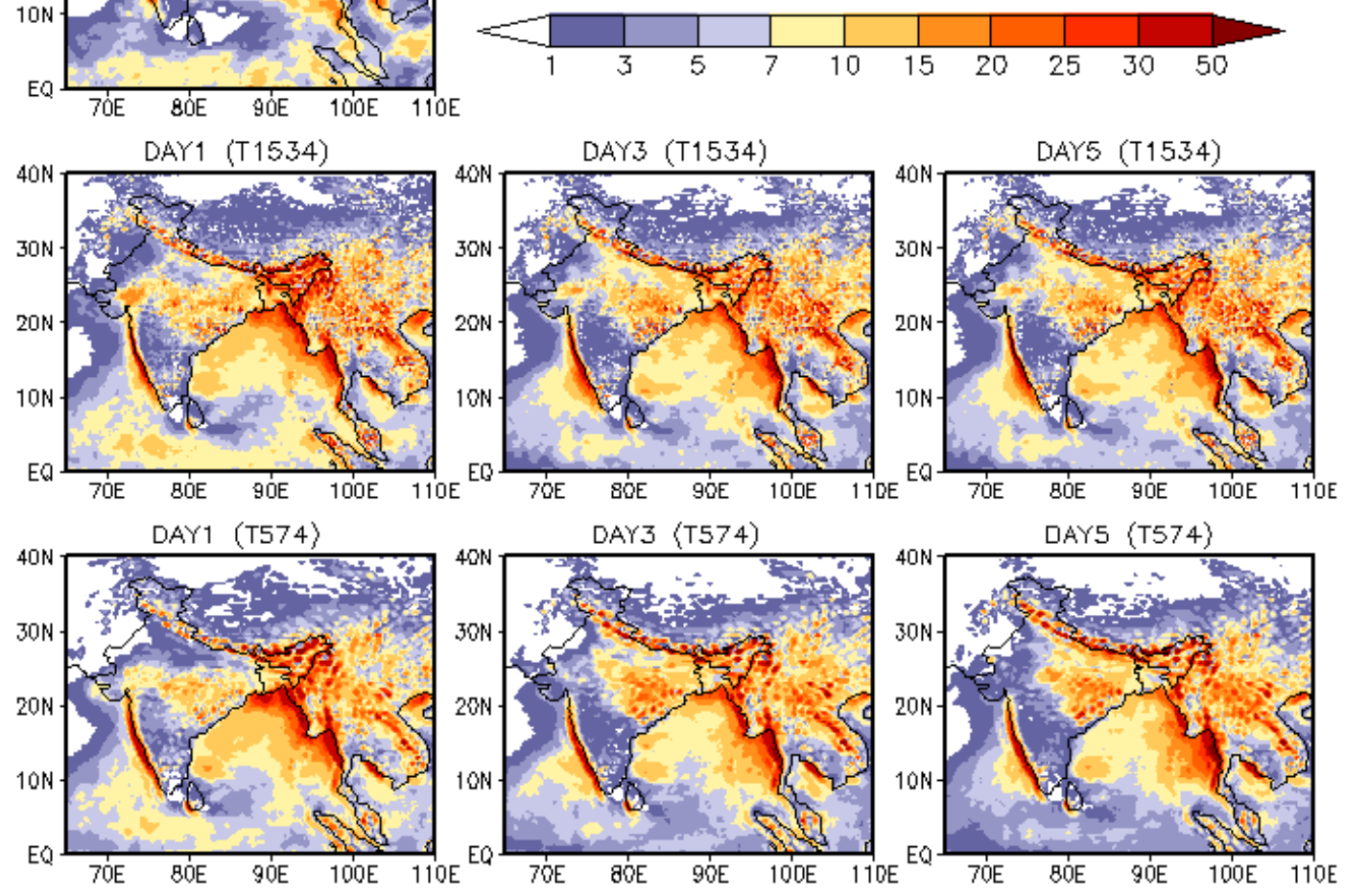

Figure 1: Mean daily precipitation for July and August 2015 from observed and model data sets 

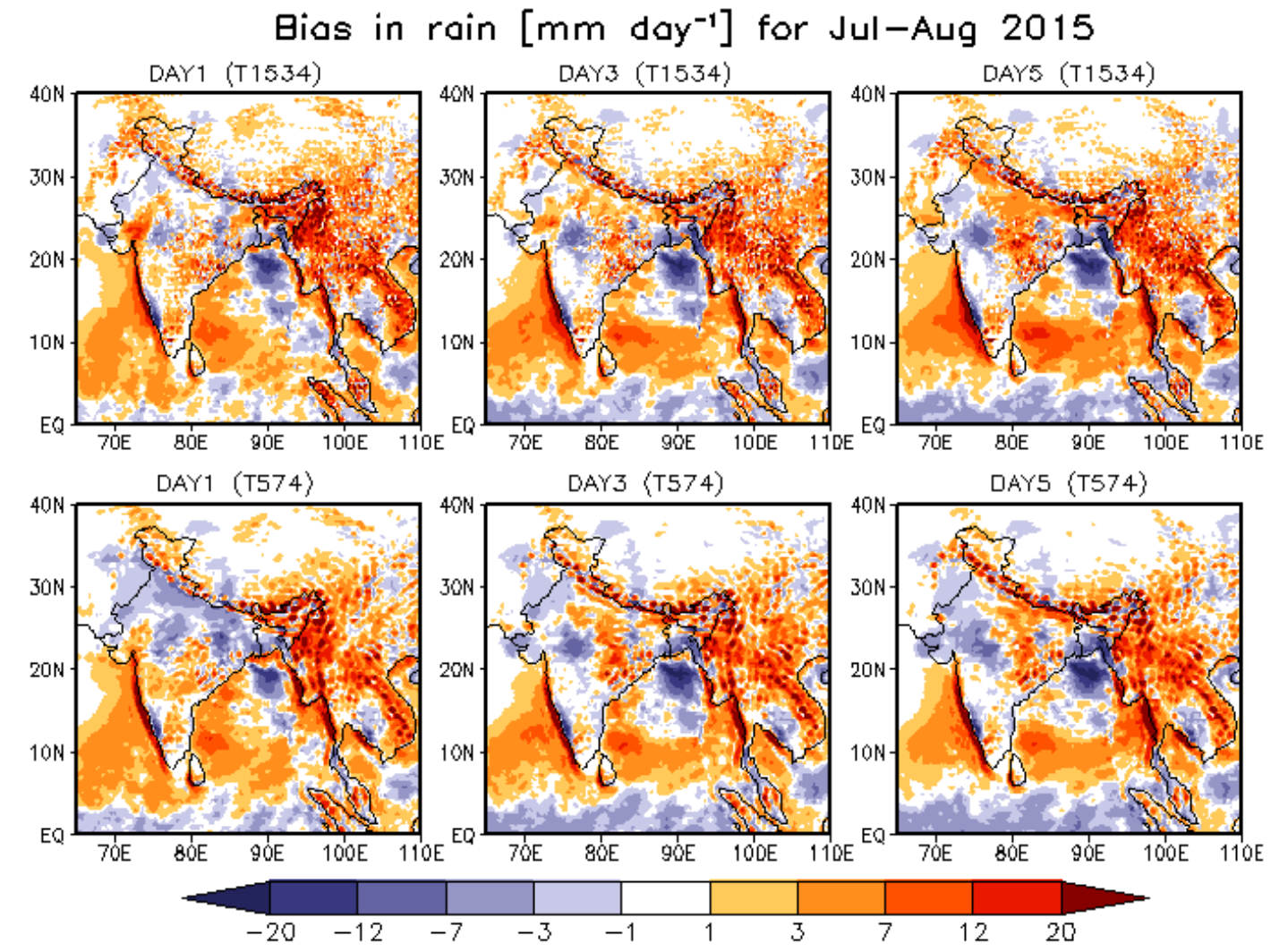

Figure 2: Spatial distributions of biases in NCEP-T1534 and NCMRWF-T574 model precipitation as compared to observation for July and August 2015 


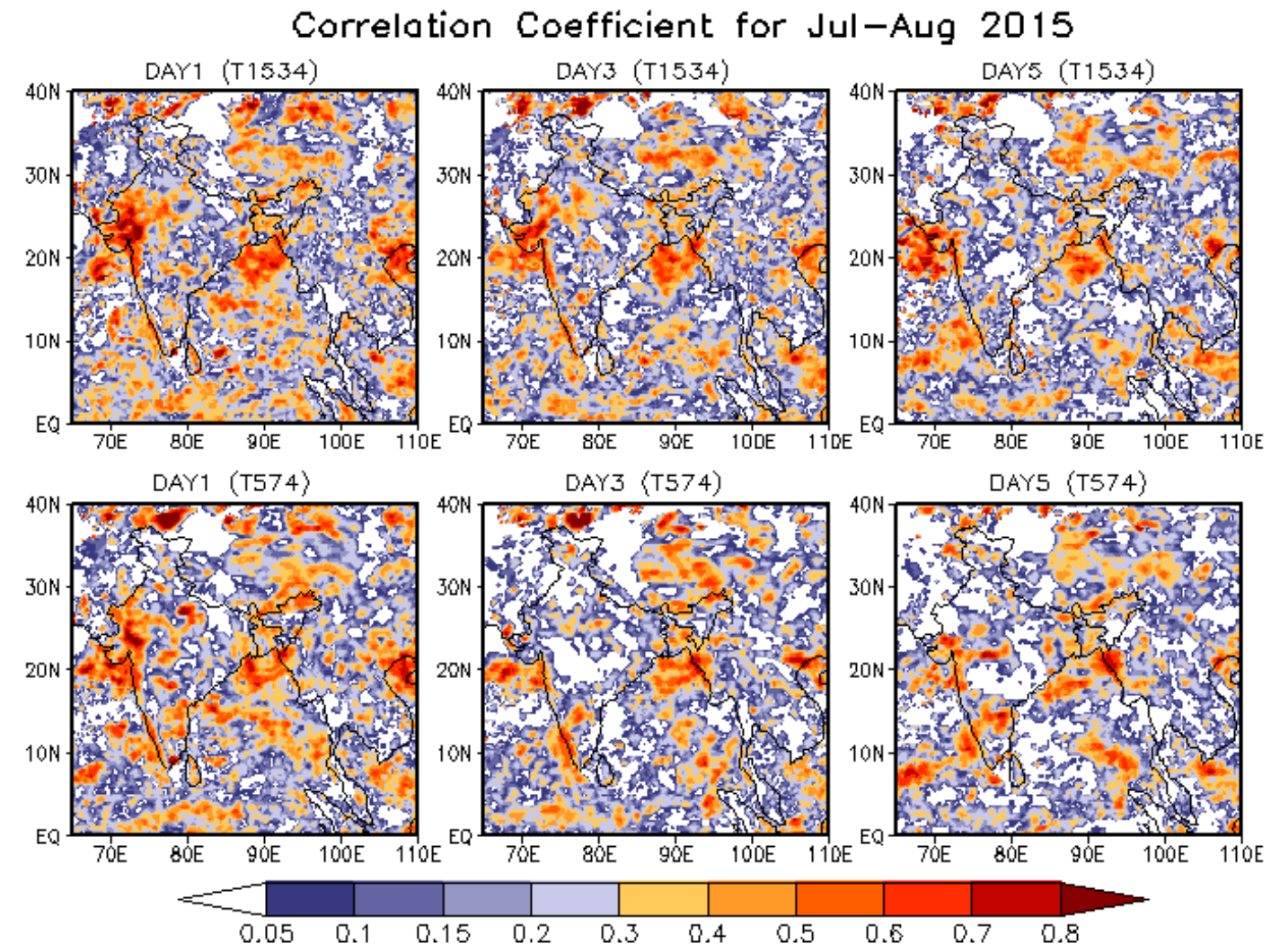

Figure 3: Spatial distributions of correlation coefficient in NCEP-T1534 and NCMRWF-T574 model precipitation as compared to observation for July and August 2015 


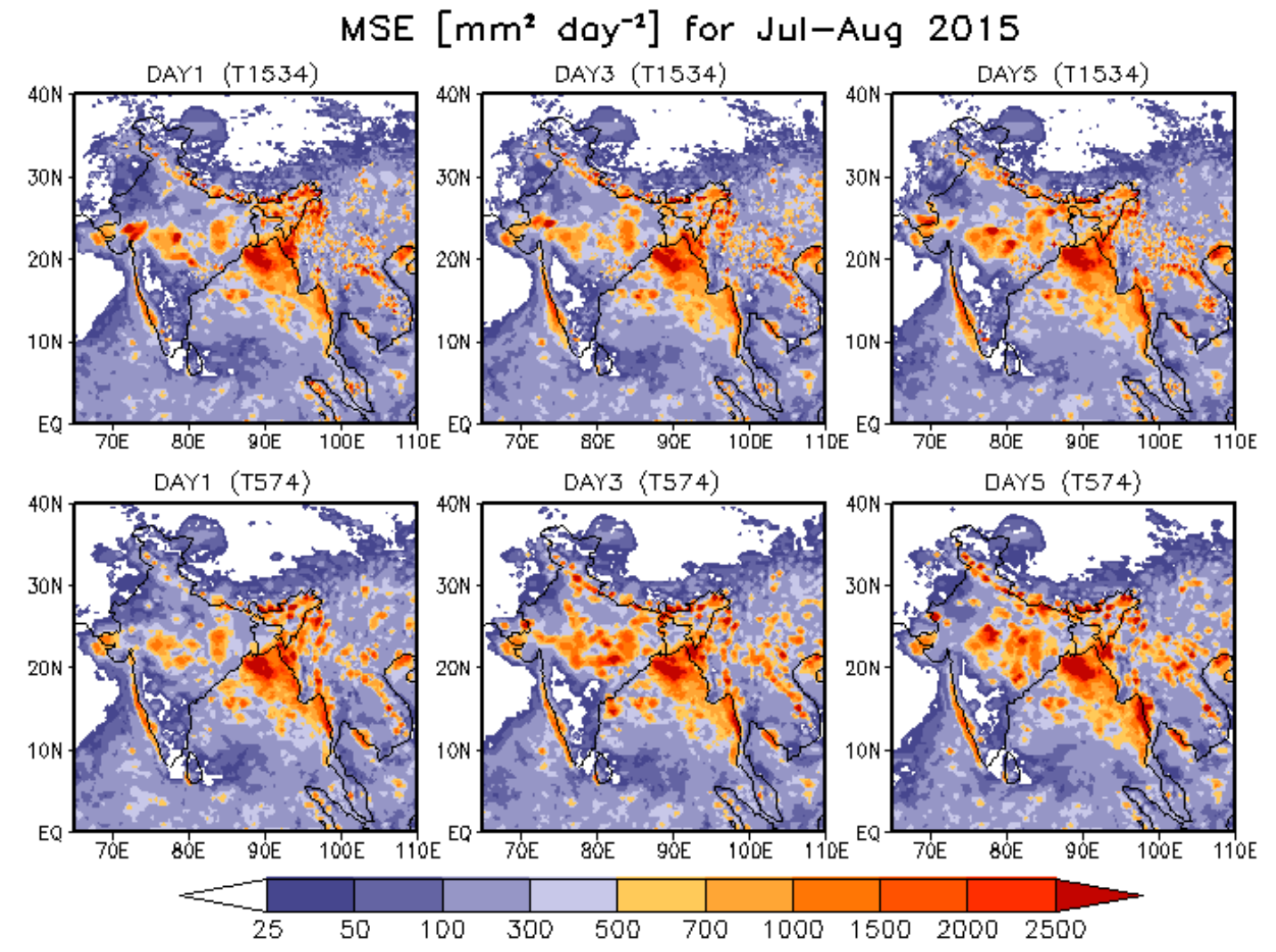

Figure 4: Spatial distributions of MSE in NCEP-T1534 and NCMRWF-T574 model precipitation as compared to observation for July and August 2015 


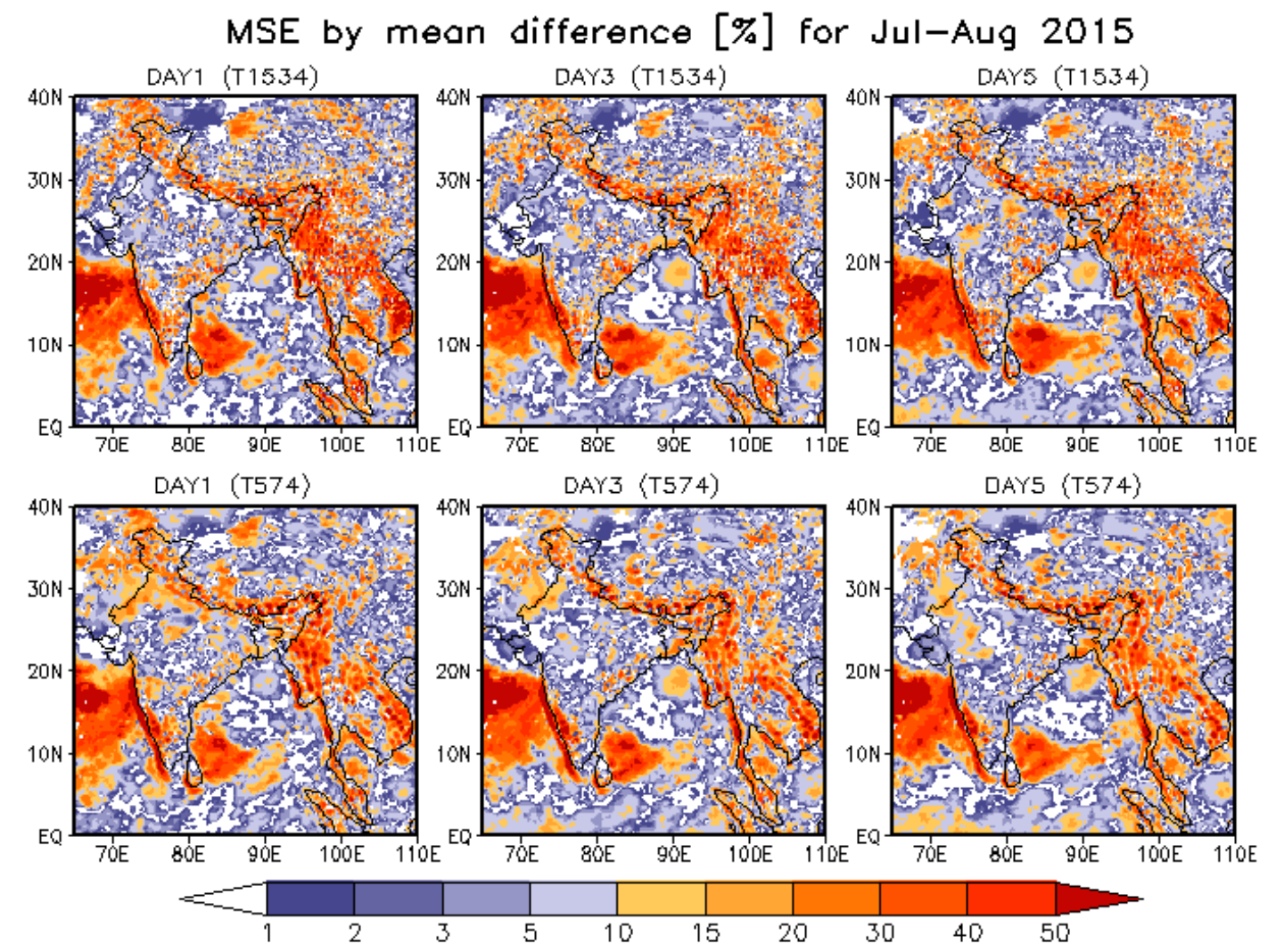

Figure 5: Spatial distributions of MSE by mean difference component in NCEP-T1534 and NCMRWF-T574 model precipitation as compared to observation for July and August 2015 


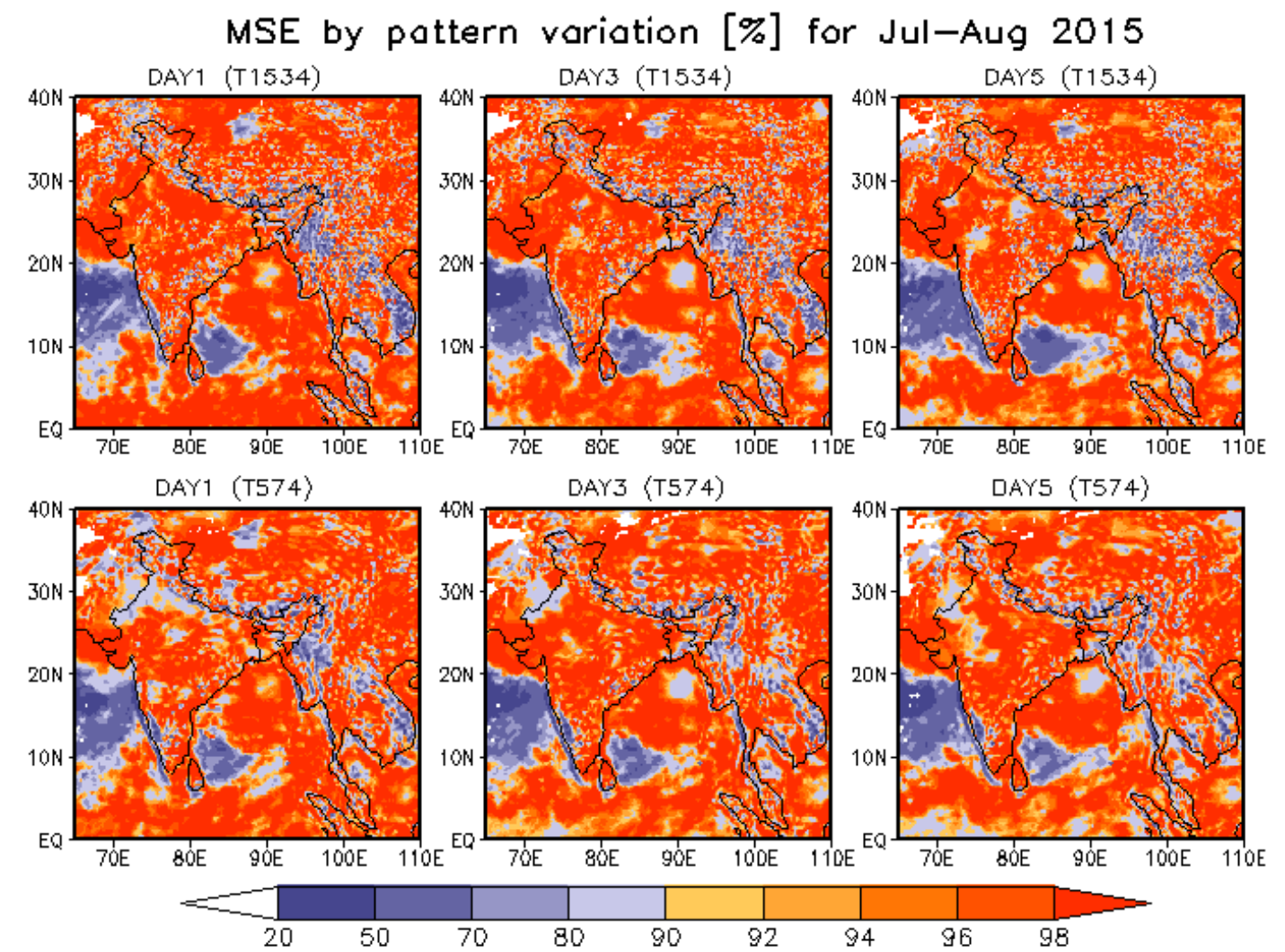

Figure 6: Spatial distributions of MSE by pattern variation component in NCEP-T1534 and NCMRWF-T574 model precipitation as compared to observation for July and August 2015

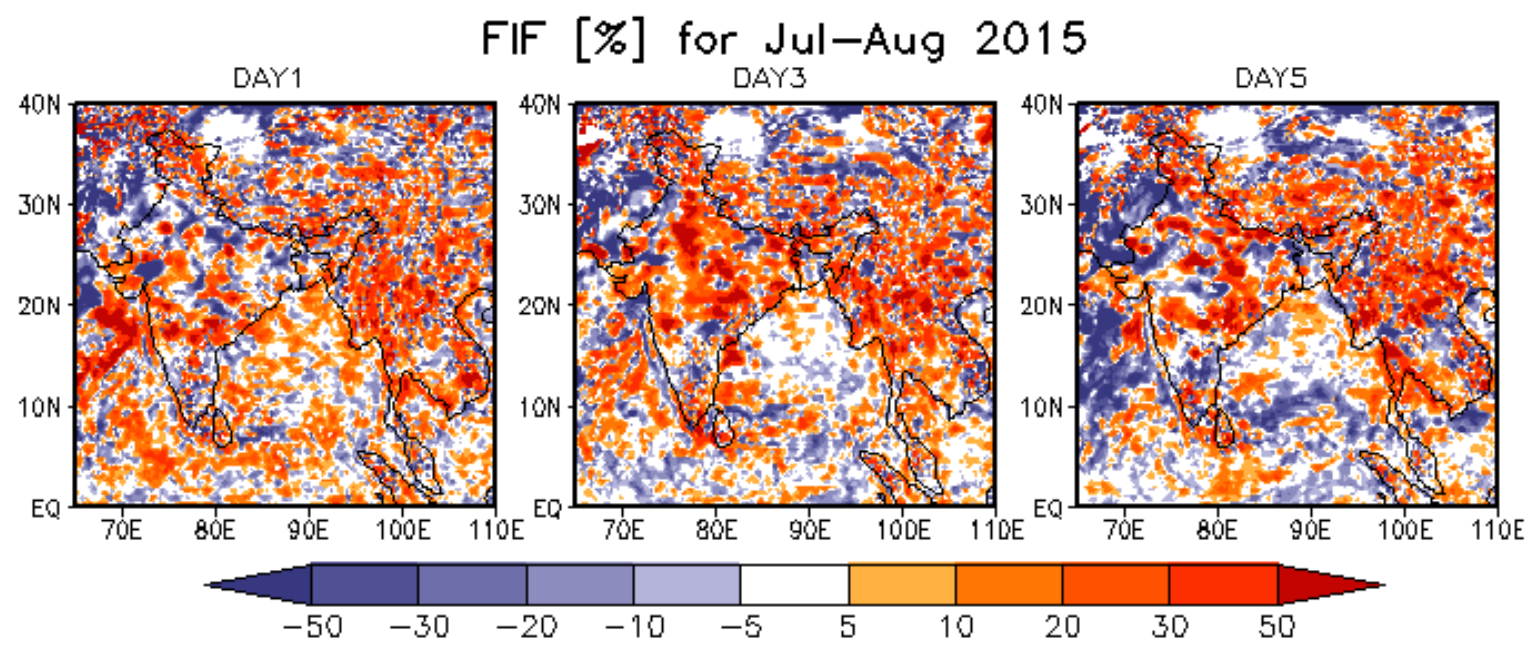

Figure 7: Forecast improvement factors for NCEP-T1534 over NCMRWF-T574 in daily precipitation forecasts during July and August 2015 

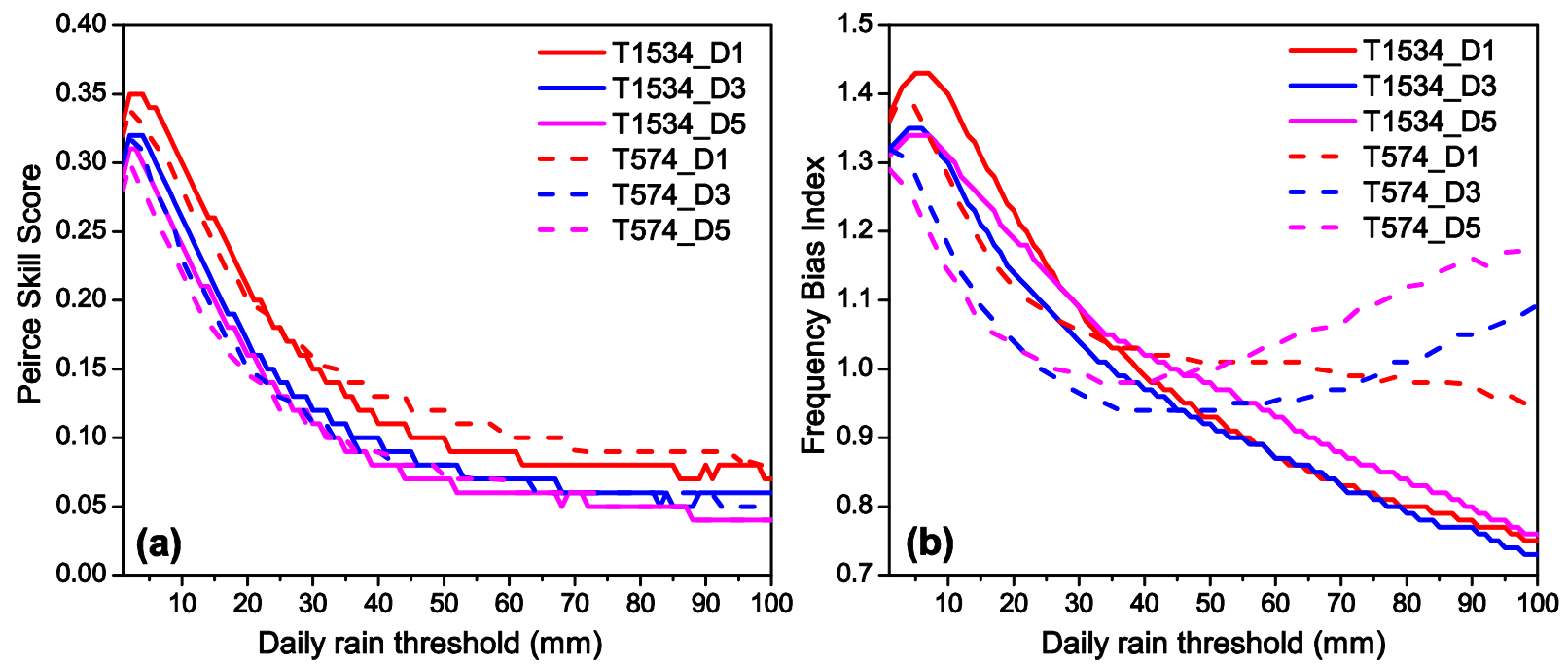

Figure 8: Variations in (a) PSS and (b) FBI in NCEP-T1534 and NCMRWF-T574 model precipitation with respect to observed data for different daily rain thresholds over the South Asia for July and August 2015
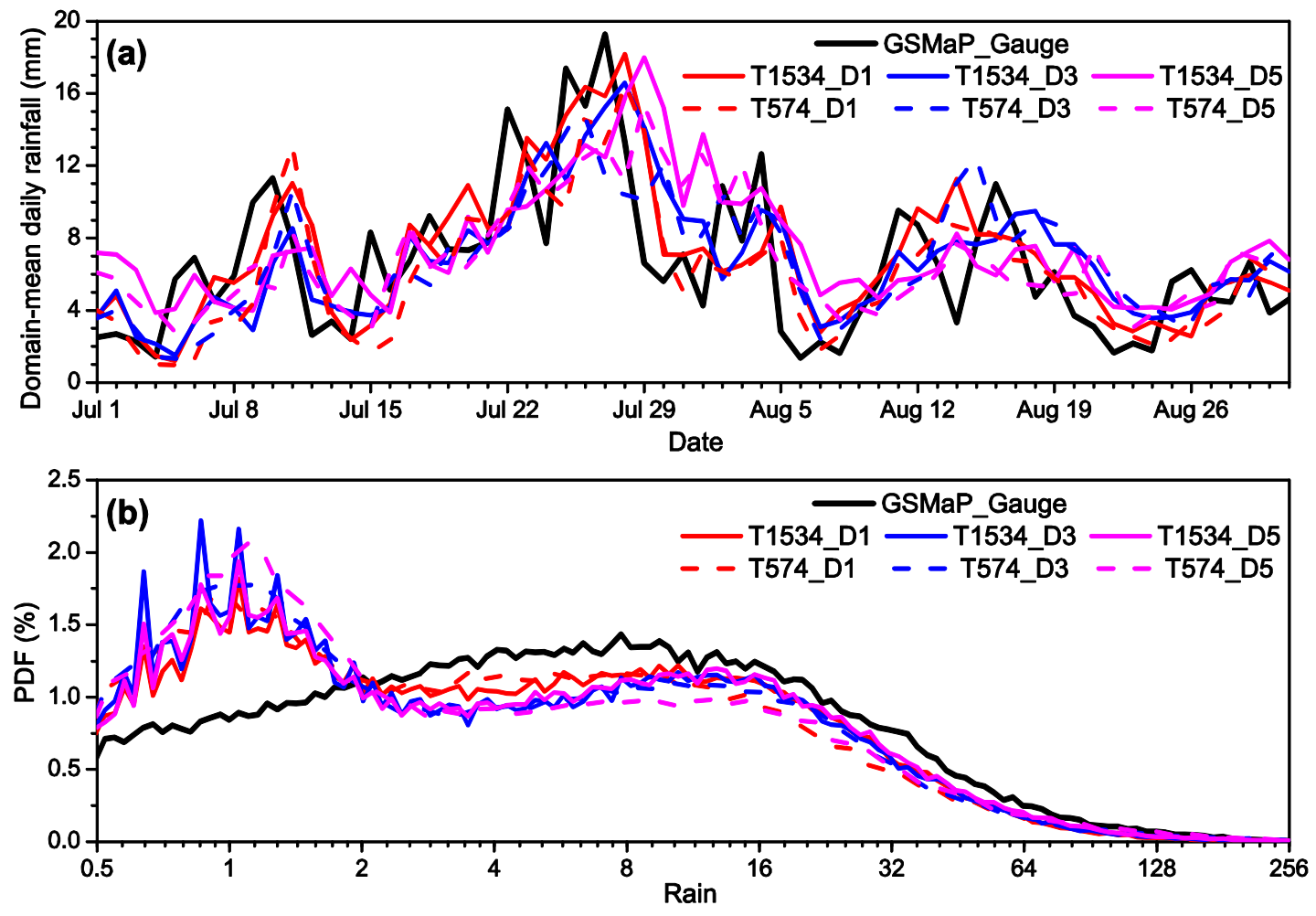

Figure 9: (a) Time-series of domain-mean daily rainfall and (b) probability distribution functions of daily precipitation over the core monsoon zone from NCEP-T1534 and NCMRWFT574 models and observed data sets for July and August 2015 